\title{
The cuticular hydrocarbons of the Triatoma sordida species subcomplex (Hemiptera: Reduviidae)
}

\author{
Gustavo Mario Calderón-Fernández, Marta Patricia Juárez/+ \\ Facultad de Ciencias Médicas, Instituto de Investigaciones Bioquímicas de La Plata, \\ Consejo Nacional de Investigaciones Científicas y Técnicas, Universidad Nacional de La Plata, La Plata, Argentina
}

\begin{abstract}
The cuticular hydrocarbons of the Triatoma sordida subcomplex (Hemiptera: Reduviidae: Triatominae) were analysed by gas chromatography and their structures identified by mass spectrometry. They comprised mostly n-alkanes and methyl-branched alkanes with one-four methyl substitutions. n-alkanes consisted of a homologous series from C21-C33 and represented 33-45\% of the hydrocarbon fraction; n-C29 was the major component. Methyl-branched alkanes showed alkyl chains from C24-C43. High molecular weight dimethyl and trimethylalkanes (from C35-C39) represented most of the methyl-branched fraction. A few tetramethylalkanes were also detected, comprising mostly even-numbered chains. Several components such as odd-numbered 3-methylalkanes, dimethylalkanes and trimethylalkanes of C37 and C39 showed patterns of variation that allowed the differentiation of the species and populations studied. Triatoma guasayana and Triatoma patagonica showed the most distinct hydrocarbon patterns within the subcomplex. The T. sordida populations from Brazil and Argentina showed significantly different hydrocarbon profiles that posed concerns regarding the homogeneity of the species. Triatoma garciabesi had a more complex hydrocarbon pattern, but it shared some similarity with T. sordida. The quantitative and qualitative variations in the cuticular hydrocarbons may help to elucidate the relationships between species and populations of this insect group.
\end{abstract}

Key words: Triatoma garciabesi - Triatoma guasayana - Triatoma patagonica - cuticle hydrocarbons - mass spectrometry

Triatomines (Hemiptera: Reduviidae: Triatominae) comprise more than 140 blood-sucking insect species; some of which are important vectors for Trypanosoma cruzi, the causative agent of Chagas disease. Triatomine species are grouped into several complexes according to their morphology, habitat and ecology (Carcavallo et al. 2000, Schofield \& Galvão 2009). The cuticular hydrocarbon pattern of the three major vectors of Chagas disease, Triatoma infestans, Rhodnius prolixus and Triatoma dimidiata, as well as those of Triatoma mazzotti and Triatoma pallidipennis, two important vectors from Mexico, has been analysed by capillary gas chromatography coupled to mass spectrometry (CGC-MS) (Juárez \& Brenner 1987, Juárez \& Blomquist 1993, Juárez et al. 2001, Calderón-Fernández et al. 2011). Triatomine hydrocarbons consist of a complex blend of saturated straight chain alkanes containing between 18-33 carbon atoms together with methyl-branched components with one-four methyl substitutions inserted into alkyl chains ranging from 25 to more than 43 carbons. Cuticle hydrocarbons participate in several aspects of insect survival and fitness. In T. infestans, the inhibition of hydrocarbon synthesis was positively correlated with a higher susceptibility to contact insecticides (Juárez 1994). In addition,

doi: 10.1590/0074-0276108062013015

Financial support: ANPCYT (PICTs 2003 01-14174, 2007 01503)

MPJ and GMCF are members of the CONICET Researcher Career.

+ Corresponding author: mjuarez@isis.unlp.edu.ar

Received 2 January 2013

Accepted 29 May 2013 pyrethroid resistance was associated with a two-fold increase in the cuticular hydrocarbon content (Pedrini et al. 2009) and reduced insecticide penetration (Juárez et al. 2010). The Triatoma sordida subcomplex includes the following four species: T. sordida, which ranges from Central Brazil throughout most parts of Paraguay and Bolivia to Central Argentina, Triatoma guasayana, which is distributed throughout Central and Northern Argentina as well as most parts of Bolivia and Paraguay, Triatoma garciabesi, which can only be found in Central and Northern Argentina and Triatoma patagonica, which is exclusively distributed in Argentina, from the Patagonia region to the central part of the country (Lent \& Wygodzinsky 1979, Jurberg et al. 1998). These species show a variable degree of association with humans and their houses. T. patagonica and T. guasayana are both mainly sylvatic, but they can form small colonies in the peridomestic habitat; also they were shown entering in human dwellings attracted to light or attacking humans in the field. T. sordida and T. garciabesi can form large colonies in peridomestic habitats (Diotaiuti et al. 1993, Wisnivesky-Colli et al. 1993). As a part of a larger project focused on detecting structural differentiation in the triatomine hydrocarbons, the aim of this work was to analyse the cuticle hydrocarbon pattern of the T. sordida subcomplex by CGC-MS analysis.

\section{MATERIALS AND METHODS}

Insects and sampling sites - Adult male and female specimens of each species were analysed. T. garciabesi and T. guasayana specimens came from Santiago del Estero (Argentina) and T. patagonica specimens came from a colony originally from Santa Fé (Argentina). Panzera et al. (1997) reported genetic differences between Brazilian 
and Argentinean populations of T. sordida. Thus, two $T$. sordida colonies were analysed; one from Rondonópolis (Brazil) and the other from Córdoba (Argentina). Insects were $\geq 1$ month old at the time of the analysis.

Cuticle lipid extraction and hydrocarbon purification - Male and female specimens were analysed separately. Samples (5 insects each) were washed with redistilled water to remove any water-soluble contaminants. Then, they were transferred to a glass tube and submerged three times in redistilled hexane (Carlo Erba Reagents, Milano, Italy) for $5 \mathrm{~min}$ each to extract the total lipids. The hexane volume was reduced under a nitrogen stream and the hydrocarbons were separated from the other lipid components by adsorption chromatography on a mini-column $[10 \mathrm{~mm}$ x $5 \mathrm{~mm}$ internal diameter (ID)] of activated Biosil A (Bio-Rad Laboratories, Richmond, CA) and eluted with redistilled hexane (4 mL).

CGC-MS analysis - The volume of the hydrocarbon extracts was reduced under a nitrogen stream and analysed by CGC-MS using a Hewlett-Packard 6890 (Hewlett Packard, Wilmington, DE) CGC to an Agilent 5975C VL (Santa Clara, CA) mass spectrometer and interfaced with an Agilent MSD Chem Station. The injection port was operated in splitless mode at $320^{\circ} \mathrm{C}$. A non-polar fused silica HP-5MS column ( $30 \mathrm{~m}$ x $0.25 \mathrm{~mm}$ ID x 0.25 $\mu \mathrm{m}$ film) was used with helium as the carrier gas at a constant flow rate of $1.5 \mathrm{~mL} / \mathrm{min}$. The oven temperature was programmed to $50^{\circ} \mathrm{C}$ for $1 \mathrm{~min}$, increased to $200^{\circ} \mathrm{C}$ at a rate of $50^{\circ} \mathrm{C} / \mathrm{min}$, further increased to $320^{\circ} \mathrm{C}$ at a rate of $3^{\circ} \mathrm{C} / \mathrm{min}$ and then finally held for $25 \mathrm{~min}$. The mass spectrometer detector was set at $70 \mathrm{eV}$ with the transfer line and the quadrupole held at $320^{\circ} \mathrm{C}$ and $150^{\circ} \mathrm{C}$, respectively. The Kovats retention index (KI) (Kovats 1965) was calculated for each hydrocarbon peak after measuring the elution times of the alkane standards run under similar conditions. The hydrocarbon peak areas were calculated for each chromatogram (HP Chem Station, Hewlett Packard) and expressed as a percentage of the total peak area. Shorthand nomenclature is used in the text and Supplementary data to identify the hydrocarbons. CXX represents the total number of carbons in the straight chain with linear alkanes denoted by $n$-CXX. The location of methyl branches was described as X-me for monomethyl alkanes, $\mathrm{x}, \mathrm{x}$-dime for dimethyl alkanes, $\mathrm{x}, \mathrm{X}, \mathrm{x}$-trime for trimethyl alkanes and $\mathrm{x}, \mathrm{x}, \mathrm{x}, \mathrm{x}$-tetrame for tetramethyl alkanes. Interpretation of the mass spectra was performed as described previously (Juárez et al. 2001, Calderón-Fernández et al. 2011).

Statistical analyses - Differences in the relative amounts of cuticle hydrocarbons between species were tested by ANOVA. Statistical significance between means was assessed by Tukey's test $(\alpha=0.05)$ using the SPSS v11.0 software (SPSS, Chicago, IL, USA).

\section{RESULTS}

About 100 components from 21-43 atoms in the carbon backbone were detected in the cuticular hydrocarbons of the T. sordida subcomplex (Fig. 1A-E, Supplementary data). Homologous series of $n$-alkanes and single-component and isomeric mixtures of meth- yl-branched alkanes with one-four methyl substitutions were found in the carbon backbone. Trace levels of $n$ alkenes were detected (data not shown) with molecular ions two mass units less than those of the corresponding $n$-alkane. Neither qualitative nor quantitative significant differences were observed between males and females in the populations examined (data not shown). Hydrocarbon component identification together with the corresponding chromatographic retention indices and characteristic mass spectral ions are shown in Supplementary data.

$n$-alkanes formed a continuous series from C21-C33. The proportion of $n$-alkanes varied from $33-45 \%$ of the total hydrocarbon fraction within the subcomplex (Supplementary data). Odd-numbered chains prevailed over even-numbered ones, with $n$-C29 predominating. The $n$-C31 chain was the second major component in T. sordida, T. guasayana and T. patagonica. In contrast, $n$-C27 was the second major component in T. garciabesi, showing a significantly lower amount in T. guasayana and $T$. patagonica $(1.86 \pm 0.27 \%$ and $1.77 \pm 0.15 \%$ respectively, $\mathrm{p}<0.05$ ) (Figs 1A-E, 2, Supplementary data).

The methyl-branched fraction was represented by a variety of mono, di, tri and tetramethylalkanes, usually present as isomeric mixtures. Most odd-numbered carbon backbones had the methyl groups inserted at oddnumbered carbon atoms, whereas even-numbered backbones had the methyl groups inserted at both odd and even-numbered carbons.

Internally-branched monomethylalkanes were found as a homologous series of minor components that eluted as isomeric mixtures with both odd and even-numbered carbon skeletons ranging from $\mathrm{C} 25-\mathrm{C} 43$ (Supplementary data). As expected, these isomeric mixtures eluted ahead of the n-alkanes with the same number of total carbons ( $\sim 73 \mathrm{KI}$ units less); the subterminally-branched components (6 and 7-me) eluted slightly closer to the corresponding straight chains ( $\sim 60 \mathrm{KI}$ units less). Terminally-branched monomethylalkanes (at branching positions C2-C5) were detected in all species of the subcomplex with alkyl chains ranging from 24-35 carbon atoms. Anteiso branching (3-me) was detected in odd and evennumbered alkyl chains, with the former prevailing as a continuous series ranging from $\mathrm{C} 25-\mathrm{C} 35$. A species-specific pattern was observed in the odd-numbered 3-methylalkanes from C27-C35 (Fig. 3, Supplementary data). In both T. guasayana and T. patagonica, 3-methylalkanes showed increasing proportions from $0.30 \pm 0.07 \%$ and $0.16 \pm 0.03 \%$ for the 3 -me $\mathrm{C} 27$ to $6.08 \pm 0.24 \%$ and $6.15 \pm$ $0.37 \%$ for the 3 -me C 33 , respectively. In contrast, the 3 -me C29 was the major component $(6.70 \pm 0.20 \%)$ followed by 3 -me C31 (1.50 $\pm 0.20 \%)$ in T. sordida, whereas both components exhibited almost similar relative amounts in $T$. garciabesi $(5.09 \pm 0.30 \%$ and $6.05 \pm 0.36 \%$ respectively). Small amounts of 3-me isomers of even-numbered chains were also found in some of the species studied. Also, 2, 4 and 5-methylalkanes were scarcely represented, except for 2-me C26 in T. garciabesi $(1.83 \pm 0.07 \%)$ and a series of 4-me components in T. sordida from Rondonópolis (4-me C28, 4-me C30 and 4-me C34 accounting for 1.23 $\pm 0.04 \%, 1.74 \pm 0.06 \%$ and $1.92 \pm 0.15 \%$ of the total hydrocarbons, respectively) (Supplementary data). 


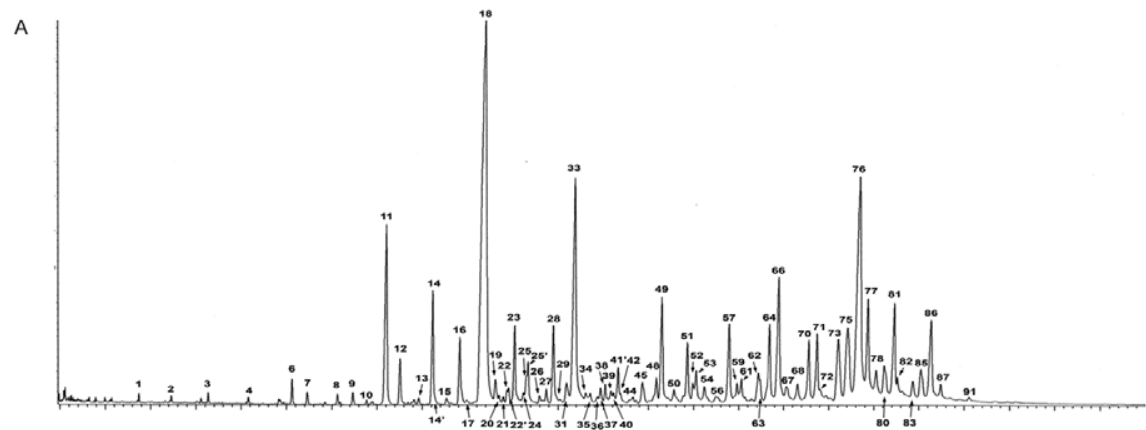

B

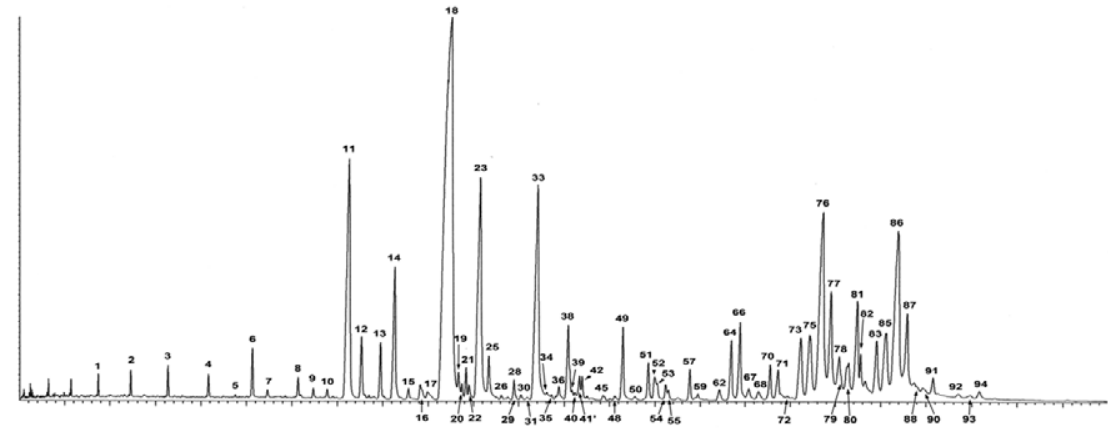

C

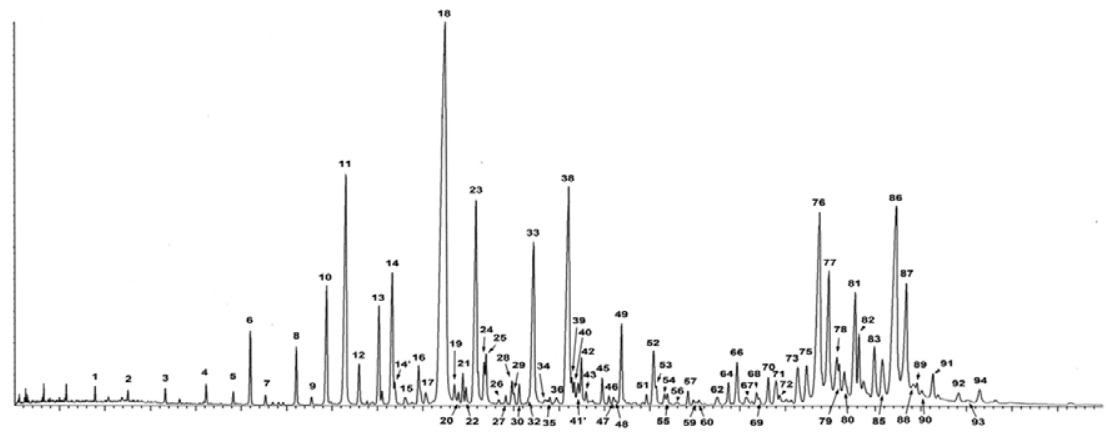

D

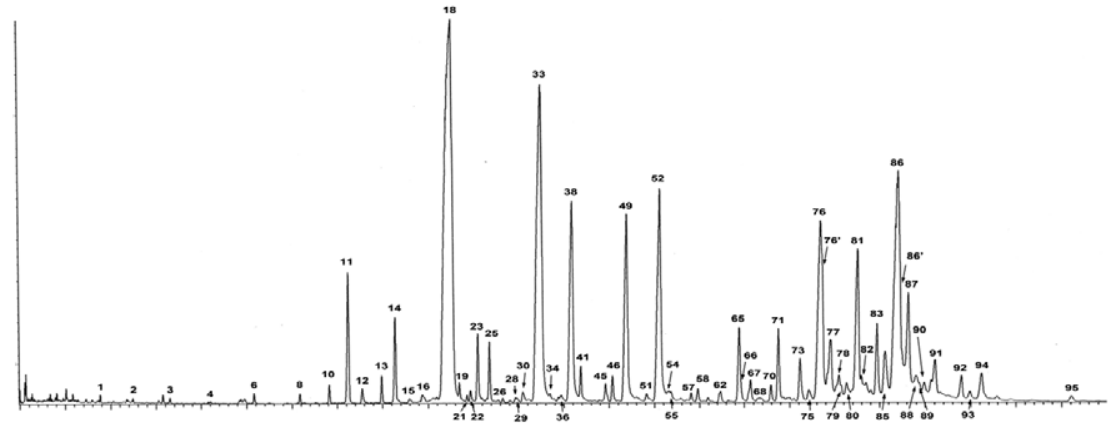

$\mathrm{E}$

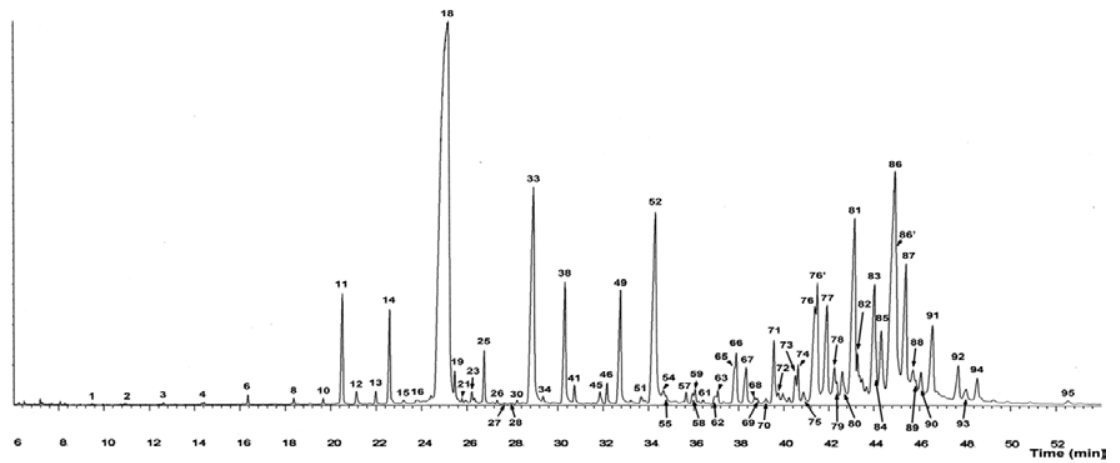

Fig 1: total ion chromatograms of male specimens of species and populations of the Triatoma sordida subcomplex. A: Triatoma sordida from Rondonópolis, Brazil; B: Triatoma sordida from Córdoba, Argentina; C: Triatoma garciabesi; D: Triatoma guasayana; E: Triatoma patagonica. Peak numbers correspond to peaks listed in Supplementary data. 
Dimethylalkanes with both odd and even-numbered carbon chains were detected with the first methyl branch positioned at either terminal $(3, \mathrm{x}-, 4, \mathrm{x}-$ and $5, \mathrm{x}-)$ or internal carbons. Terminal dimethylalkanes mostly had 7 or more methylene groups separating methyl branches; 3 methylene groups usually separated the methyl groups in internally-branched dimethylalkanes (Supplementary data). The relative amounts of several dimethylalkanes showed significant differences among species (Fig. 4, Supplementary data). For example, the 13,23- and 11,21dime C35 isomers (KI 3562) represented $2.07 \pm 0.15 \%$ and $1.12 \pm 0.07 \%$ of the total hydrocarbons in the $T$. sordida specimens (Rondonópolis and Córdoba respectively), decreased to $0.38 \pm 0.03 \%$ in $T$. garciabesi and was undetectable in both T. guasayana and T. patagonica. Similarly, the relative amounts of the dimethyl isomers of C37 (KI 3757) varied from $2.95 \pm 0.13 \%$ in T. sordida (Rondonópolis) and $2.12 \pm 0.08 \%$ in T. sordida (Córdoba) to $1.09 \pm 0.07 \%$ in $T$. garciabesi and further diminished to $0.25 \pm 0.06 \%$ and $0.21 \pm 0.06 \%$ in $T$. guasayana and T. patagonica, respectively. Conversely, the latter two species had significantly higher relative amounts of the 5,X-dime isomers of C37 and C39 (KI 3782 and 3982 respectively), whereas these components were undetectable or present in trace amounts in T. sordida and T. garciabesi. A series of 3, $\mathrm{x}$-dime isomers $(\mathrm{x}=7,9,11,13,15$ and 17) were detected in odd-numbered carbon chains from C27-C39 (Supplementary data). The 3,x-dime isomers of C37 (KI 3805) and C39 (KI 4005) were the predominant components, eluting with a KI of 100-95 units less than $n$-alkanes with the same total number of carbon atoms (Supplementary data). 3, $\mathrm{x}$-dime isomers of C39 were significantly different among species, particularly between T. sordida populations (Fig. 4). Dimethylalkanes with even-numbered carbon skeletons were represented by terminal and internal components of 30-40 carbon atoms in the alkyl chain. Terminal components consisted of low amounts of 4,8, 4,16 and 4,18-dime isomers detected in carbon chains C30-C40; only the 4,x-dime isomers of $\mathrm{C} 38$ were found in the four species studied, ranging from $0.42 \pm 0.02 \%$ to $1.34 \pm 0.09 \%$ of the total hydrocarbons (Supplementary data). Dimethylalkanes with both methyl branches positioned internally included the $6,10,12,22$, 14,18 and 16,20 isomers with straight backbones from 3440 carbons (Supplementary data). 6,10-dimethylalkanes co-eluted with the trimethyl components with the same number of carbons in the alkyl chain (Supplementary data), but they were identified by the presence of a strong ion doublet at $\mathrm{m} / \mathrm{z}$ 98/99 together with a significant ion at $m / z 169$ corresponding to the presence of the methyl groups at carbons 6 and 10, respectively.

Trimethylalkanes with both odd and even-numbered carbon skeletons from 29-41 carbons were found throughout the subcomplex (Supplementary data). Internallybranched trimethyl alkanes usually had three methylene groups between the first and the second methyl branches and five methylene groups between the second and the third branch $(3+5$ pattern); to a lesser extent, a $3+3$ pattern was also detected. Thus, mixtures of $11,15,21,11,17$, $21,13,17,21,13,17,23$ and 13,19,23 components prevailed in odd-numbered carbon backbones from C31-C41, while $12,16,22,12,18,22,14,18,24$ and 14,18,22 components were found in even-numbered backbones from C34-C40. Even-numbered chains with methyl branches positioned at odd carbons were also detected, such as 11,17,21-trime C32, 11,15,21-trime C34 and 13,17,23 plus 13,19,23-trime C36. Internal trimethyl isomers of C37 and C39 were the major methyl-branched components of the subcom-

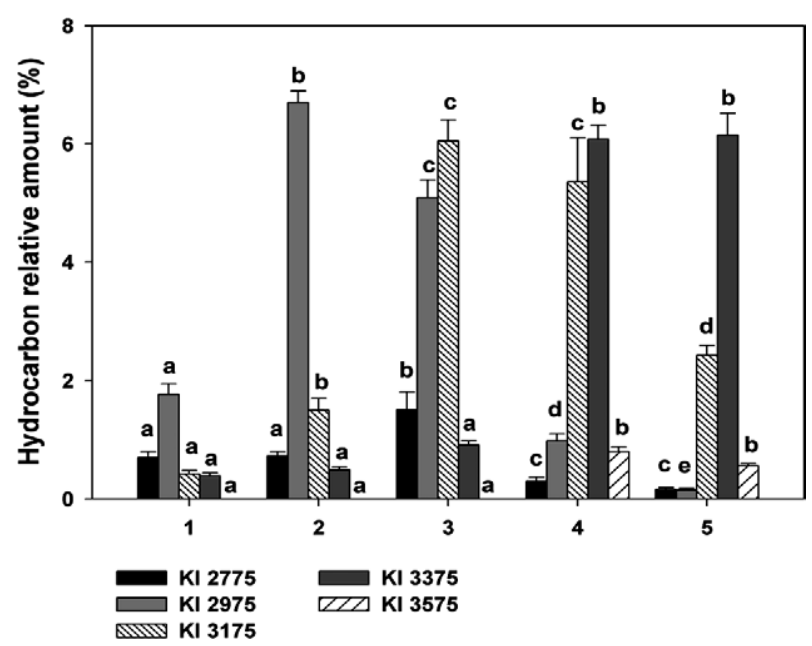

Fig 3: variation of odd-numbered 3-methylalkanes among species and populations of the Triatoma sordida subcomplex. 1: T. sordida (Rondonópolis, Brazil); 2: T. sordida (Córdoba, Argentina); 3: Triatoma garciabesi; 4: Triatoma guasayana; 5: Triatoma patagonica. Different letters in hydrocarbon amount indicate significant differences ( $\mathrm{p}$ $<0.05$ ) among groups by Tukey's test. Kovats index (KI) 2775: 3-me C27; KI 2975: 3-me C29; KI 3175: 3-me C31; KI 3375: 3-me C33; KI

3575: 3-me C35.
Fig 2: variation of major $n$-alkanes among species and populations of the Triatoma sordida subcomplex. 1: T. sordida (Rondonópolis, Brazil); 2: T. sordida (Córdoba, Argentina); 3: Triatoma garciabesi; 4: Triatoma guasayana; 5: Triatoma patagonica. Different letters in hydrocarbon amount indicate significant differences $(\mathrm{p}<0.05)$ among groups by Tukey's test. Kovats index (KI) 2700: $n$-C27; KI 2900: $n$-C29; KI 3100: $n$-C31; KI 3300: $n$-C33.

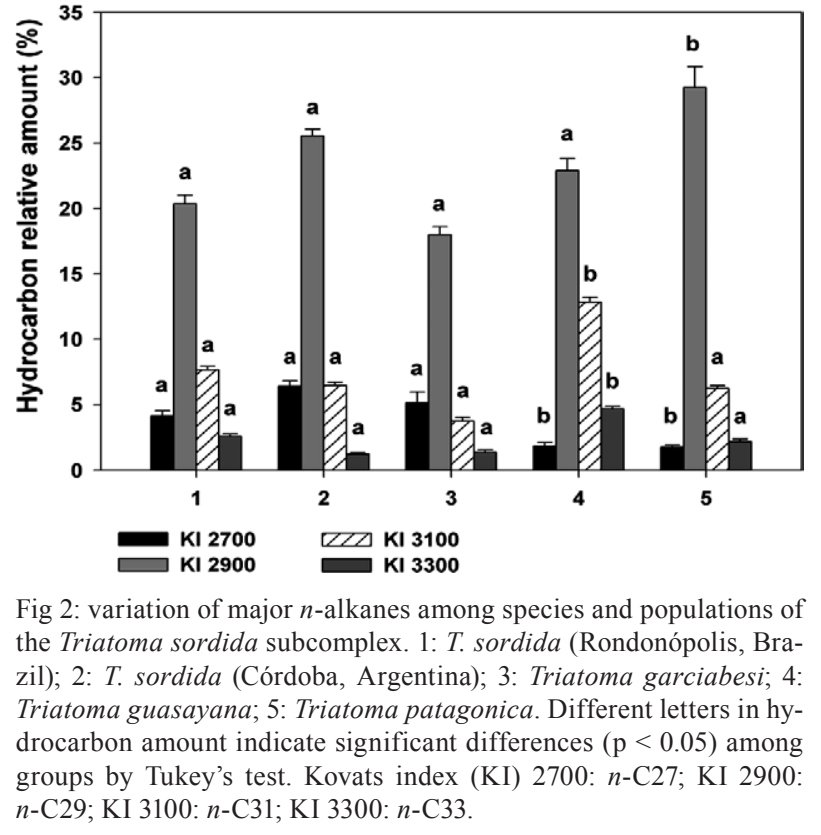


plex, showing a specific pattern of variation. The relative amounts of internal trimethyl isomers of C37 (KI 3780) decreased from $10.00 \pm 0.60 \%$ in T. sordida (Rondonópolis) to $2.66 \pm 0.15 \%$ in $T$. patagonica, showing the most significant difference between the two T. sordida populations, as well as between T. guasayana and T. patagonica (Fig. 5, Supplementary data). In contrast, the relative amounts of internal trimethyl isomers of C39 (KI 3980) increased from $2.48 \pm 0.11 \%$ in T. sordida (Rondonópolis) to $10.19 \pm 0.40 \%$ in T. patagonica, showing significant differences among T. sordida from Brazil, T. sordida from Argentina and T. garciabesi (Fig. 5, Supplementary data). Terminal trimethylalkanes included the 2,6,10 isomers of C34, C36 and C38; 3,7,x- and 3,11,15 isomers of $\mathrm{C} 35, \mathrm{C} 37$ and C39 and traces of 4,8,x isomers of C36 and C38 (Supplementary data). The 2,6,10 isomers co-eluted with the internally-branched odd-numbered monomethylalkanes of C35, C37 and C39, respectively (Supplementary data). 3,x, $\mathrm{x}$-trimethyl branching was detected in odd-numbered alkyl chains from C35-C39. Similarly to $2, \mathrm{x}, \mathrm{x}$-trimethyl alkanes, they co-eluted with or close to the internally-branched monomethylalkanes of C36, C38 and C40, respectively (Supplementary data).

Few tetramethylalkanes were detected in the species subcomplex. A complete series was found in T. patagonica with small amounts of 4,8,12,16-tetrame isomers of C34, C36 and C38. Also, the 3,7,11,15-tetrame of C35 (KI 3653) was detected throughout the species subcomplex, peaking at $1.52 \pm 0.12 \%$ in $T$. sordida (Rondonópolis).

\section{DISCUSSION}

Insect cuticular hydrocarbons often occur as complex blends of straight-chain and methyl-branched components, with saturated and unsaturated alkyl chains ranging from about 21 to more than 50 carbons (Howard $\&$ Blomquist 2005, Blomquist 2010). Among other functions, cuticle hydrocarbons have been shown to participate in protecting insects against desiccation, microbial attack and insecticide penetration (Juárez 1994, Napolitano \& Juárez 1997, Pedrini et al. 2009) and also play a key role in chemical communication as species, mate and nestmate recognition cues (Blomquist \& Bagnères 2010). In triatomines and several other insect groups, the hydrocarbon pattern is mainly under genetic control (Juárez \& Calderón-Fernández 2007, Wicker-Thomas \& Chertemps 2010). Thus, the expression of specific cuticular hydrocarbons reflects the expression of genes coding for hydrocarbon biosynthetic enzymes. Accordingly, triatomine cuticle hydrocarbons have been used as characters to address taxonomic analysis either at genera, species and populations (Juárez et al. 2000, CalderónFernández et al. 2005, 2011, 2012).

Complex mixtures of hydrocarbons were detected in the four species of the T. sordida subcomplex. These mixtures included straight chain alkanes and a variety of mono, di, tri and tetramethylalkanes comprising alkyl chains from 21-43 carbons. In triatomines, an abundant number of tetramethylalkanes were found in R. prolixus (Juárez et al. 2001) and T. dimidiata (Calderón-Fernández et al. 2011). However, they were not detected in $T$. infestans, a phylogenetically similar species (Juárez \& Blomquist 1993). Similar to their morphological features, the species of this subcomplex shared a similar hydrocarbon pattern that can be distinguished from the hydrocarbon pattern found in other triatomine species. Therefore, mostly quantitative differences in the cuticular hydrocarbon composition were detected, together with some minor qualitative differences. Several hydrocar-

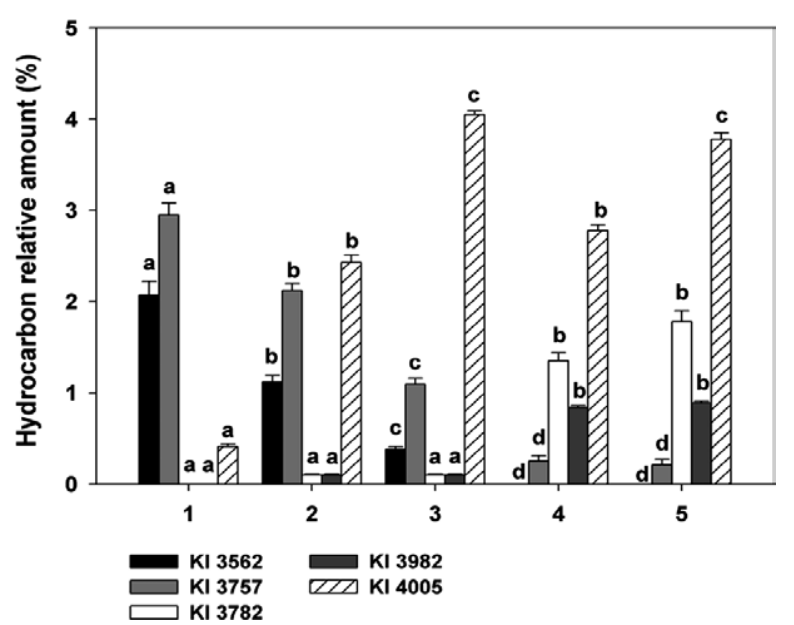

Fig 4: variation of some dimethylalkanes among species and populations of the Triatoma sordida subcomplex. 1: T. sordida (Rondonópolis, Brazil); 2: T. sordida (Córdoba, Argentina); 3: Triatoma garciabesi; 4: Triatoma guasayana; 5: Triatoma patagonica. Different letters in hydrocarbon amount indicate significant differences $(\mathrm{p}<0.05)$ among groups by Tukey's test. Kovats index (KI) 3562: 11, x and 13,xdime C35; KI 3757: 11,x, 13,x and 15,x-dime C37; KI 3782: 5,x-dime C37; KI 3982: 5,x-dime C39; KI 4005: 3,x-dime C39.

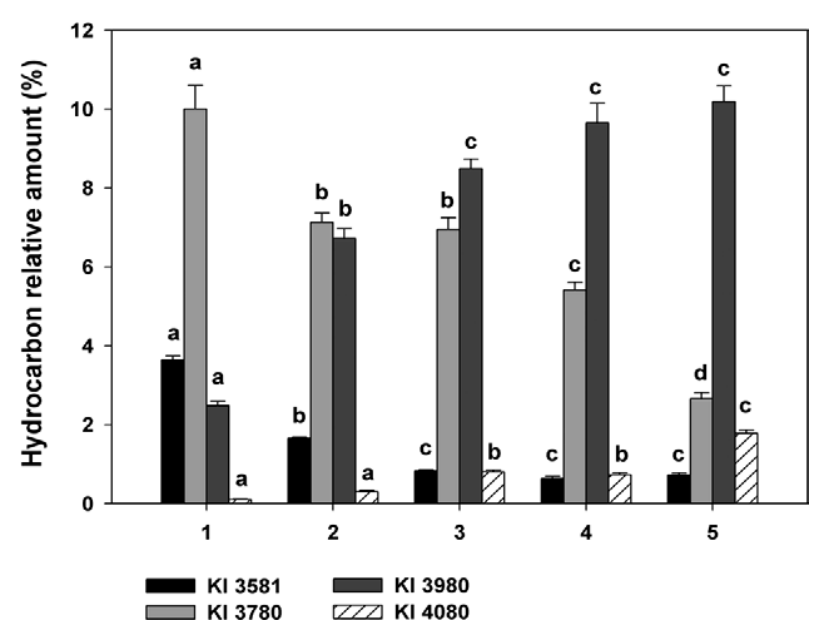

Fig 5: variation of some trimethylalkanes among species and populations of the Triatoma sordida subcomplex. 1: T. sordida (Rondonópolis, Brazil); 2: T. sordida (Córdoba, Argentina); 3: Triatoma garciabesi; 4: Triatoma guasayana; 5: Triatoma patagonica. Different letters in hydrocarbon amount indicate significant differences ( $\mathrm{p}$ $<0.05$ ) among groups by Tukey's test. Kovats index (KI) 3581: 11, x, $\mathrm{x}$ and 13,x,x-trime C35; KI 3780: 13,x,x and 15,x,x-trime C37; KI 3980: 13,x,x and 15,x,x-trime C39; KI 4080: 14,x,x and 16,x,x-trime C40. 
bons showed patterns of variation that helped to clearly discriminate the species and populations analysed in this work. T. guasayana and T. patagonica showed simpler cuticular hydrocarbon profiles with smaller amounts (or absence) of several mono, di and trimethyl alkanes from C25-C35 (Fig. 1D, E, Supplementary data). In contrast, they showed relatively larger amounts of some terminally-branched components (3-me C33, 3-me C35 and 5,x-dime isomers of C37 and C39) that were present in minor amounts or absent in T. garciabesi and T. sordida. T. garciabesi showed a more complex chromatographic profile, especially in terms of the number of hydrocarbon peaks (Supplementary data). However, the profile is clearly similar to that of T. sordida, regardless of its origin (Fig. 1A-C). T. garciabesi showed no evident qualitative differences, but significant quantitative differences in several hydrocarbons, such as 2-me C28, 3-me C27, 3-me C29 etc (Supplementary data). The analysis of the two geographically distant $T$. sordida populations revealed that they had significant quantitative differences in several components such as the 4-me series from C28$\mathrm{C} 34$, the 4,x-dime series from $\mathrm{C} 30-\mathrm{C} 34$, the 3-me C29 and $\mathrm{C} 31$, the trimethyl isomers of C35, C37 and C39 and others (Figs 3-5, Supplementary data). Some of these differences were equivalent to those found between $T$. garciabesi and $T$. sordida, thus suggesting that $T$. sordida is too heterogeneous to be considered a single species. Interestingly, previous analyses using a variety of techniques suggested that $T$. sordida consisted of at least two cryptic species (Panzera et al. 1997, Noireau et al. 1998).

After the classification proposed by Lent and Wygodzinsky (1979), the taxonomic status of the T. sordida species subcomplex has been scarcely studied, probably because these species were considered secondary vectors without relevance in transmitting Chagas disease. Furthermore, the few studies performed using morphological, morphometric or chromosomal characters have led to distinct and sometimes contradictory results. $T$. garciabesi was synonymised with $T$. sordida some years after its description (Lent \& Wygodzinsky 1979); later on, it was revalidated as a valid species (Jurberg et al. 1998). The analysis of several metric variables of head and male genitalia completely differentiated T. sordida from both T. guasayana and T. patagonica, but failed to differentiate the latter two species (Gorla et al. 1993). In contrast, cytogenetic and enzyme electrophoresis studies clearly differentiated T. guasayana from both T. $p a$ tagonica and T. sordida. These analyses also separated T. sordida into two groups (Brazil and Argentina), but a scarce differentiation was achieved between $T$. sordida populations from the wet and dry regions of Argentina (those of the dry region were formerly described as T. garciabesi) (Panzera et al. 1997). More recently, an extensive phylogenetic analysis of the Triatominae subfamily based on molecular data placed the species into a different triatomine group (Hypsa et al. 2002). Thus, current evidence suggests that the taxonomy of the subcomplex should be revised, especially regarding the relationship between $T$. guasayana and T. patagonica, as well as the intraspecific variation of the so-called "T. sordida." The use of cuticular hydrocarbons as taxonomic characters might help to elucidate the precise relationship between these species, as well as their intraspecific variability. This knowledge would provide valuable information to Chagas disease control programs in their efforts to monitor and control triatomine populations.

\section{ACKNOWLEDGEMENTS}

To Raúl Stariolo (Servicio Nacional de Chagas, Córdoba, Argentina) and Dr Francois Noireau (in memoriam) (Institut de Recherche pour le Développement, La Paz, Bolivia), for the provision of insects.

\section{REFERENCES}

Blomquist GJ 2010. Structure and analysis of insect hydrocarbons. In GJ Blomquist, A-G Bagnères, Insect hydrocarbons: biology, biochemistry and chemical ecology, Cambridge University Press, Cambridge, p. 19-34.

Blomquist GJ, Bagnères A-G 2010. Introduction: history and overview of insect hydrocarbons. In GJ Blomquist, A-G Bagnères, Insect hydrocarbons: biology, biochemistry and chemical ecology, Cambridge University Press, Cambridge, p. 3-18.

Calderón-Fernández GM, Girotti JR, Juárez MP 2011. Cuticular hydrocarbons of Triatoma dimidiata (Hemiptera: Reduviidae): intraspecific variation and chemotaxonomy. J Med Entomol 48: 262-271.

Calderón-Fernández GM, Girotti JR, Juárez MP 2012. Cuticular hydrocarbon pattern as a chemotaxonomy marker to assess intraspecific variability in Triatoma infestans, a major vector of Chagas disease. Med Vet Entomol 26: 201-209.

Calderón-Fernández GM, Juárez MP, Ramsey J, Salazar-Schettino PM, Monroy MC, Ordoñez R, Cabrera M 2005. Cuticular hydrocarbon variability among Triatoma dimidiata (Hemiptera: Reduviidae) populations from Mexico and Guatemala. J Med Entomol 42: $780-788$.

Carcavallo RU, Jurberg J, Lent H, Noireau F, Galvão C 2000. Phylogeny of the Triatominae (Hemiptera: Reduviidae). Proposals for taxonomic arrangements. Entomol Vect 7: 1-99.

Diotaiuti L, Loiola CF, Falcão PL, Dias JCP 1993. The ecology of Triatoma sordida in natural environments in two different regions of the state of Minas Gerais, Brazil. Rev Inst Med Trop S Paulo 35: 237-245.

Gorla DE, Jurberg J, Catalá SS, Schofield CJ 1993. Systematics of Triatoma sordida, T. guasayana and T. patagonica (Hemiptera, Reduviidae). Mem Inst Oswaldo Cruz 88: 379-385.

Howard RW, Blomquist GJ 2005. Ecological, behavioral and biochemical aspects of insect hydrocarbons. Annu Rev Entomol 50: 371-393.

Hypsa V, Tietz DF, Zrzavý J, Rego RO, Galvão C, Jurberg J 2002. Phylogeny and biogeography of Triatominae (Hemiptera: Reduviidae): molecular evidence of a New World origin of the Asiatic clade. Mol Phylogenet Evol 23: 447-457.

Juárez MP 1994. Inhibition of cuticular lipid synthesis and its effect on insect survival. Arch Insect Biochem Physiol 25: 177-191.

Juárez MP, Blomquist GJ 1993. Cuticular hydrocarbons of Triatoma infestans and Triatoma mazzottii. Comp Biochem Physiol B 106: 667-674.

Juárez MP, Blomquist GJ, Schofield CJ 2001. Hydrocarbons of Rhodnius prolixus, a Chagas disease vector. Comp Biochem Physiol B 129: 733-746.

Juárez MP, Brenner RR 1987. Hydrocarbons of Triatoma pallidipennis. Comp Biochem Physiol B 87: 233-239.

Juárez MP, Calderón Fernández GM 2007. Cuticular hydrocarbons of triatomines. Comp Biochem Physiol A 147: 711-730. 
Juárez MP, Fernández R, Schofield CJ, Dujardin JP 2000. Intergeneric comparison of epicuticular hydrocarbons in Triatomineae. Res Rev Parasitol 60: 121-127.

Juárez MP, Pedrini N, Girotti JR, Mijailovsky SJ 2010. Pyrethroid resistance in Chagas disease vectors: the case of Triatoma infestans cuticle. Resistance Pest Manag News 19: 59-61.

Jurberg J, Galvão C, Lent H, Monteiro F, Macedo Lopes C, Panzera F, Pérez R 1998. Revalidação de Triatoma garciabesi Carcavallo, Cichero, Martínez, Prosen \& Ronderos, 1967 (Hemiptera: Reduviidae). Entomol Vect 5: 107-122.

Kovats E 1965. Gas chromatographic comparison of organic substances in the retention index system. Adv Chromat 1: 229-247.

Lent H, Wygodzinsky P 1979. Revision of the Triatominae (Hemiptera, Reduviidae) and their significance as vector of Chagas disease. Bull Am Mus Nat Hist 163: 123-520.

Napolitano R, Juárez MP 1997. Entomopathogenous fungi degrade epicuticular hydrocarbons of $T$. infestans. Arch Biochem Biophys 344: 208-214.

Noireau F, Gutierrez T, Zegarra M, Flores R, Brenière F, Cardozo L, Dujardin JP 1998. Cryptic speciation in Triatoma sordida
(Hemiptera: Reduviidae) from the Bolivian Chaco. Trop Med Int Health 3: 364-372.

Panzera F, Hornos S, Pereira J, Cestau R, Canale D, Diotaiuti L, Dujardin JP, Pérez R 1997. Genetic variability and geographic differentiation among three species of Triatomine bugs (HemipteraReduviidae). Am J Trop Med Hyg 57: 732-739.

Pedrini N, Mijailovsky SJ, Girotti JR, Stariolo R, Cardozo RM, Gentile A, Juárez MP 2009. Control of pyrethroid-resistant Chagas disease vectors with entomopathogenic fungi. PLoS Negl Trop Dis 3: e434.

Schofield CJ, Galvão C 2009. Classification, evolution and species groups within the Triatominae. Acta Trop 110: 88-100.

Wicker-Thomas C, Chertemps T 2010. Molecular biology and genetics of hydrocarbon production. In GJ Blomquist, A-G Bagnères, Insect hydrocarbons: biology, biochemistry and chemical ecology, Cambridge University Press, Cambridge, p. 53-74.

Wisnivesky-Colli C, Gürtler RE, Solarz ND, Schweigmann NJ, Pietrokovsky SM, Alberti A, Flo J 1993. Dispersive flight and house invasion by Triatoma guasayana and Triatoma sordida in Argentina. Mem Inst Oswaldo Cruz 88: 27-32. 


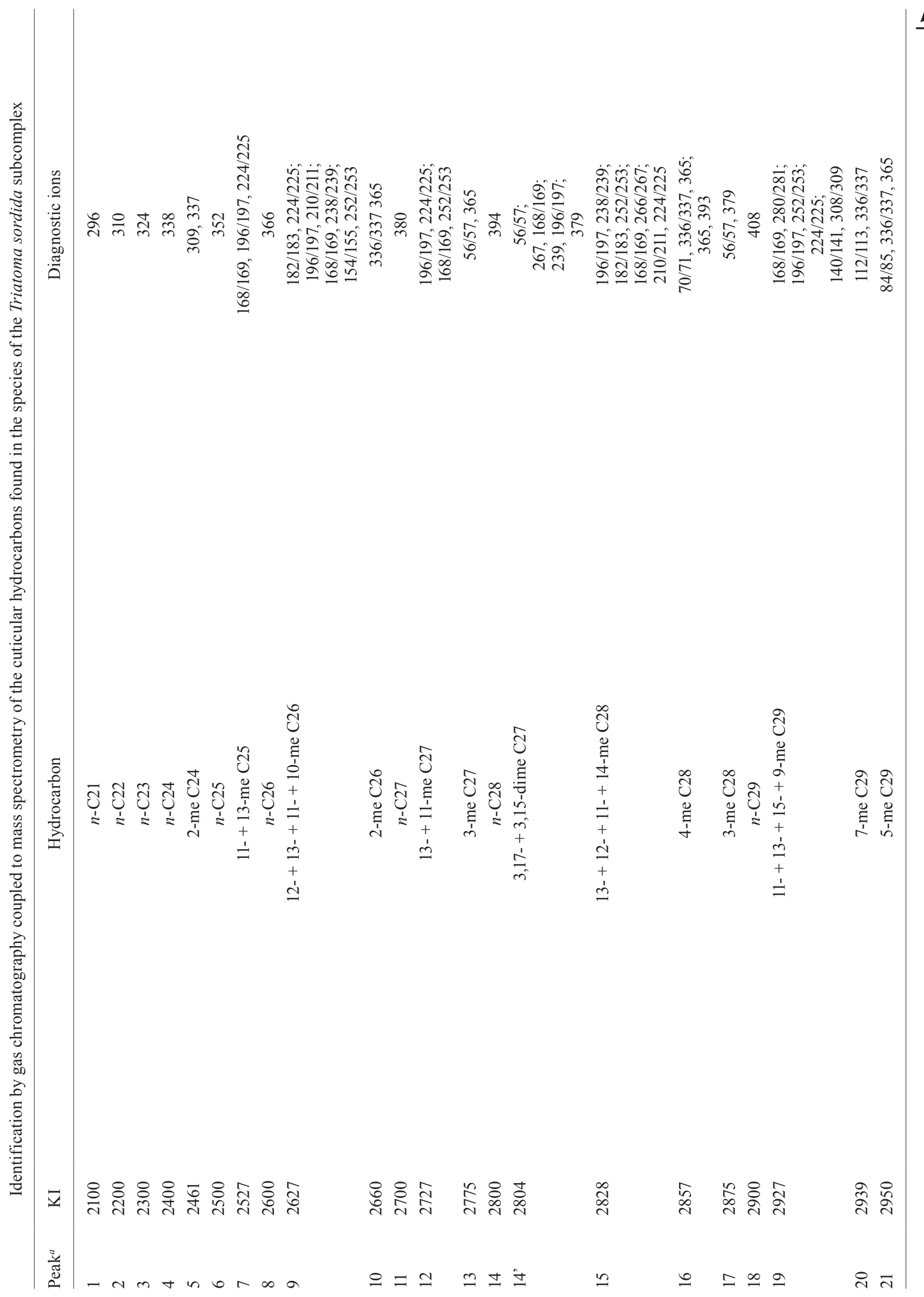




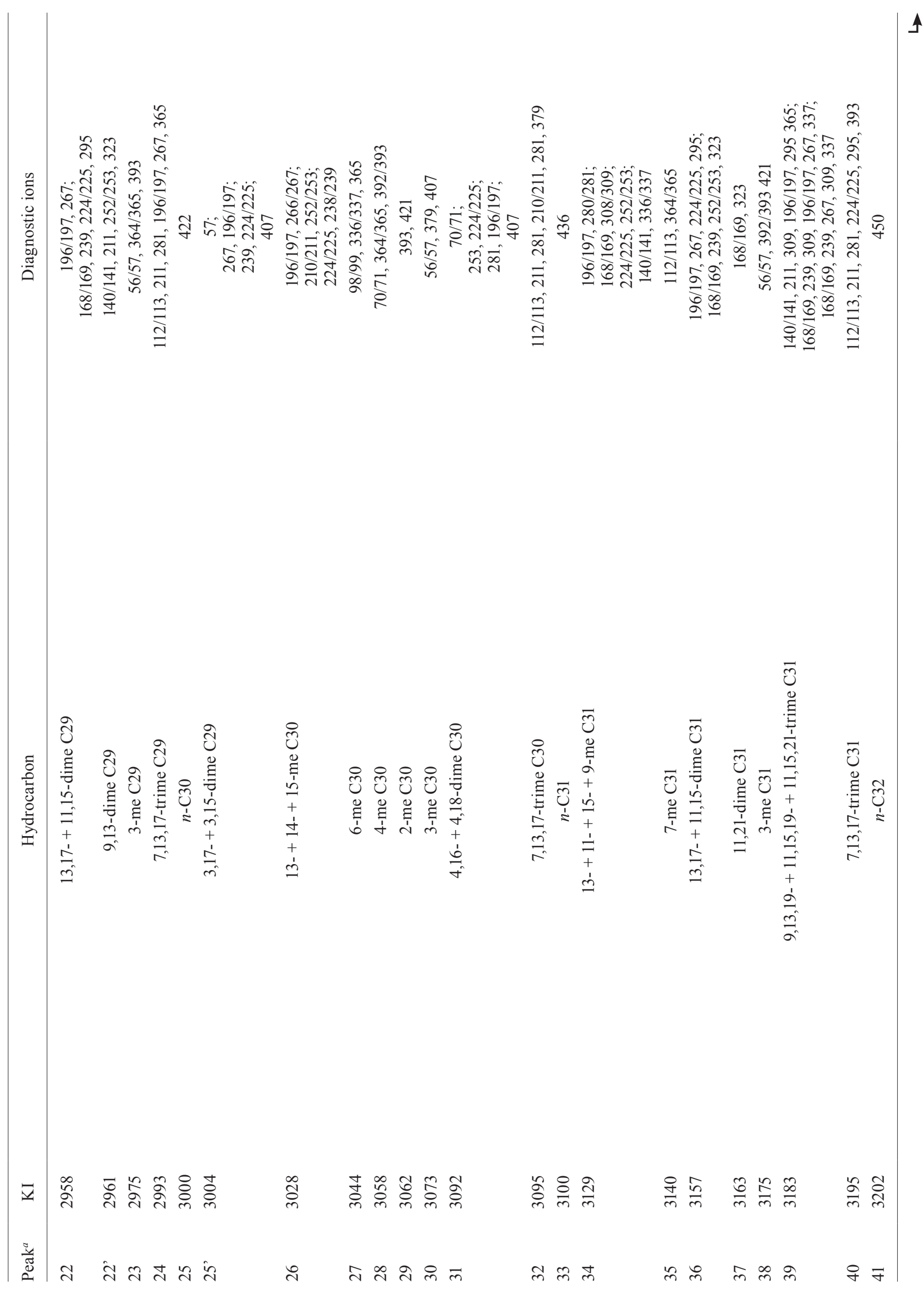




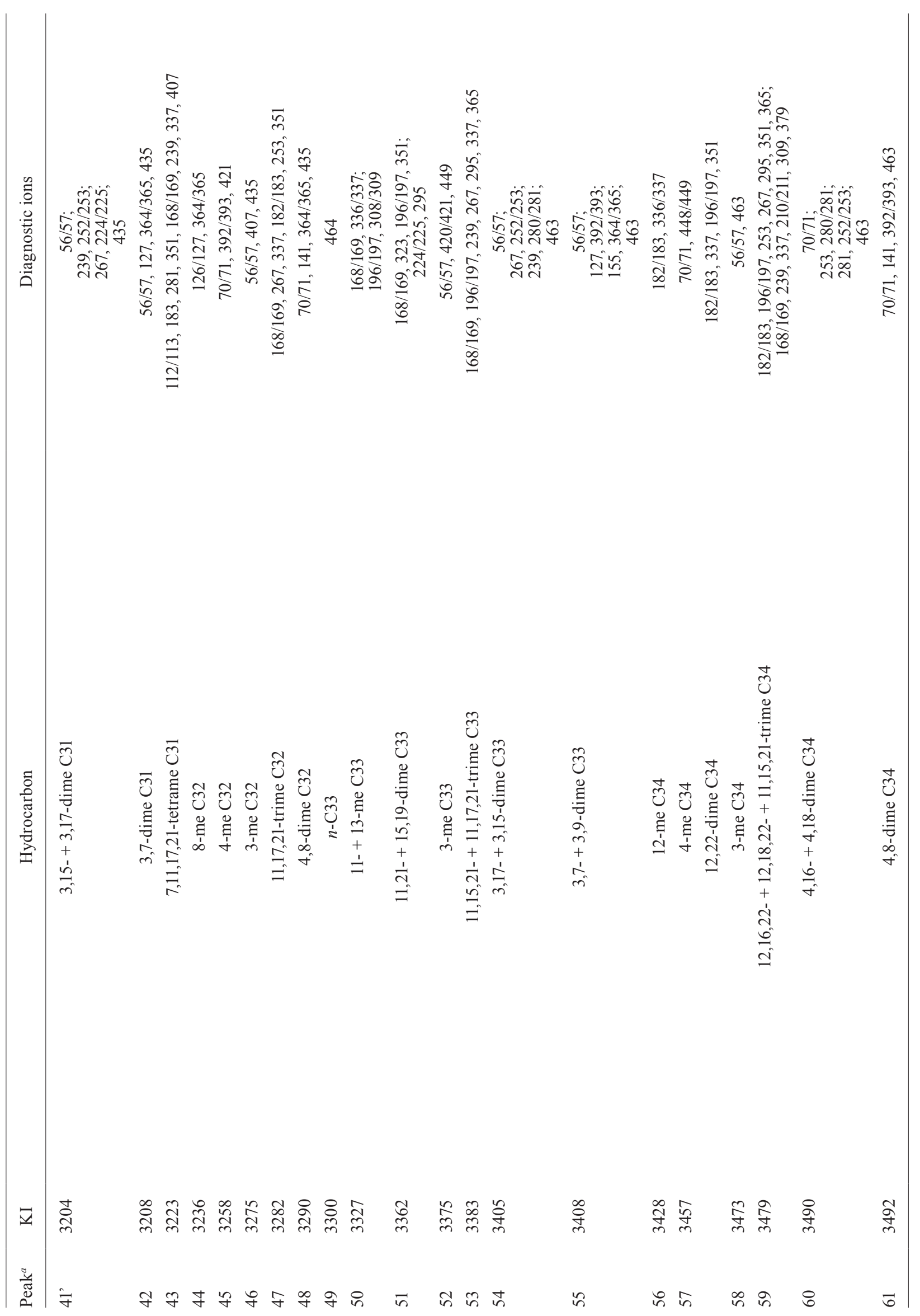




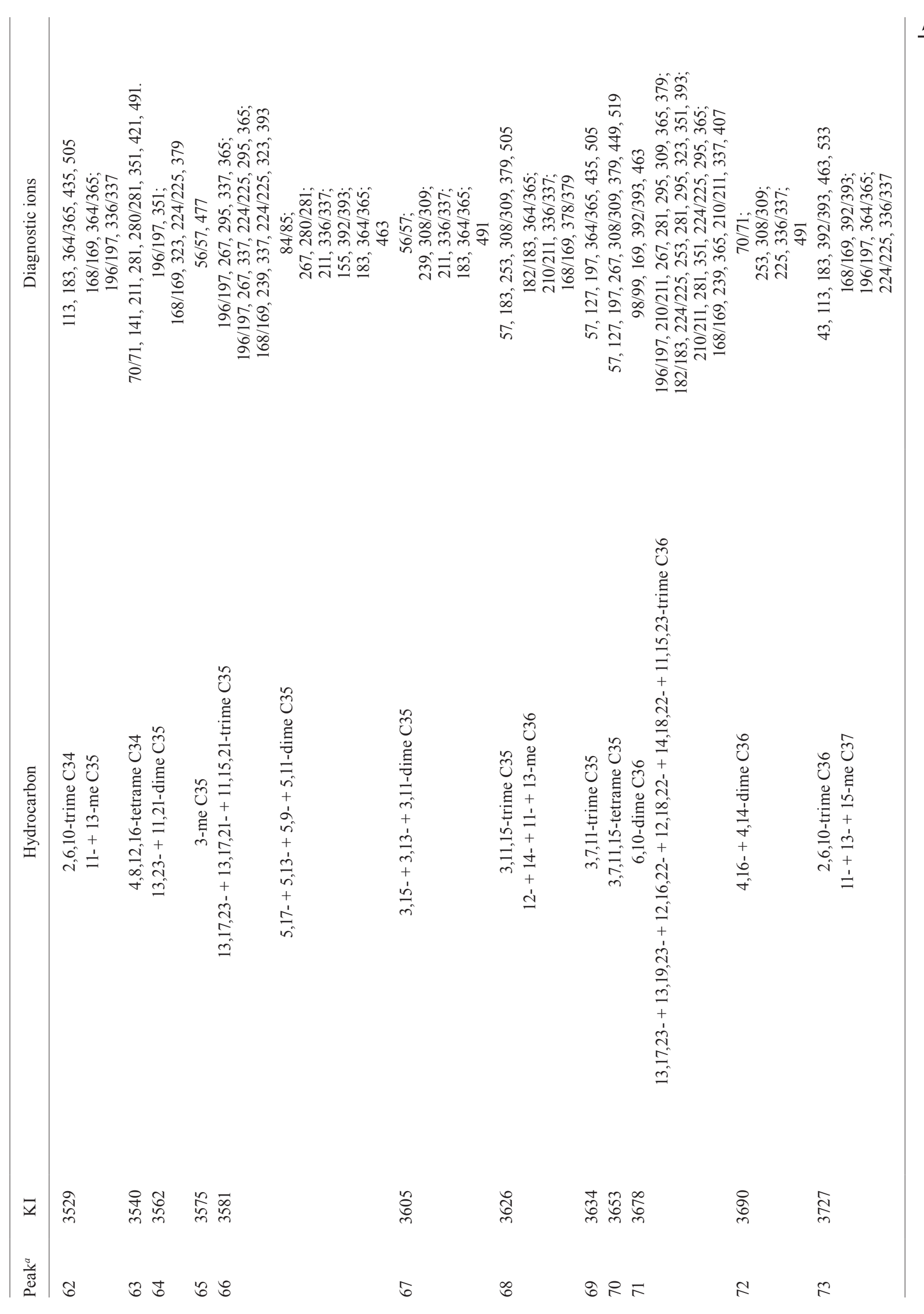




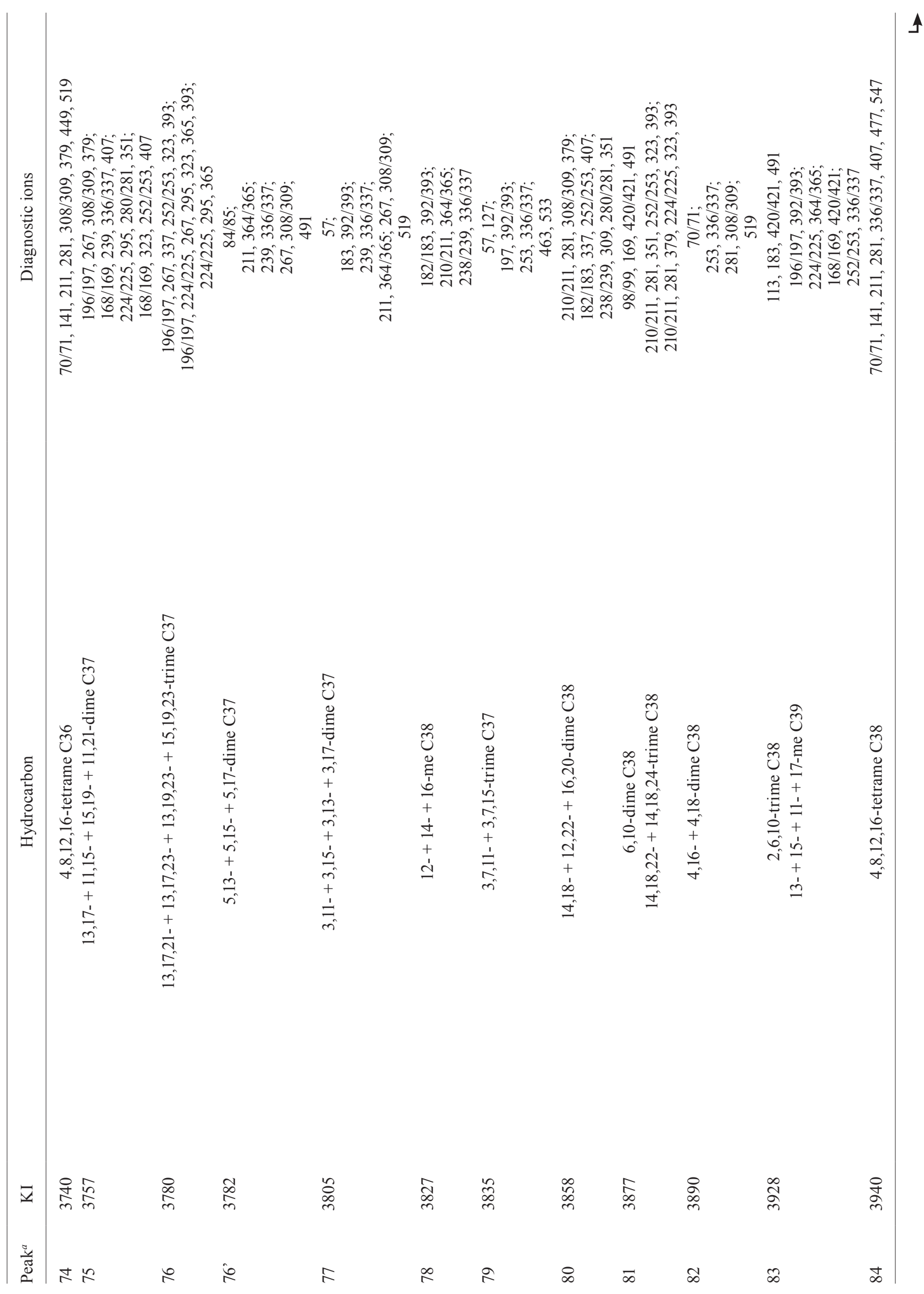




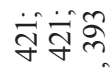

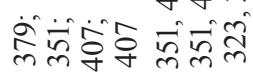

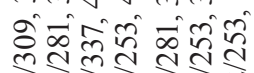

के

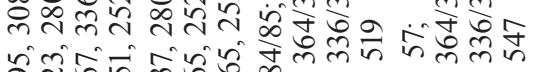

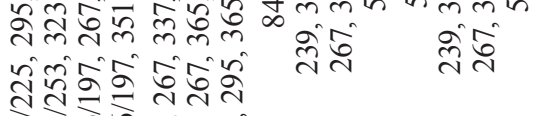

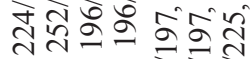

๖워ำ $\ddot{\vec{\gamma}} \ddot{\bar{\gamma}} 2$

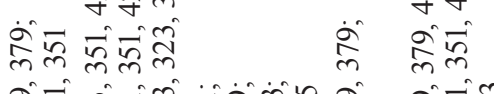

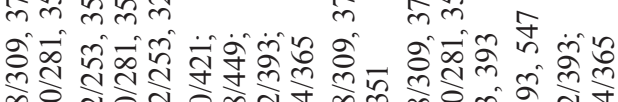

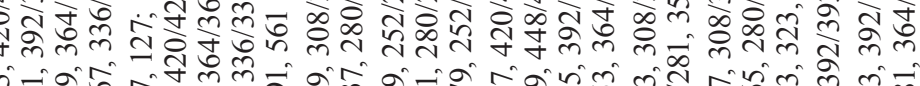

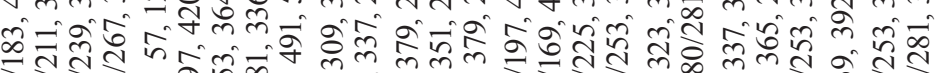

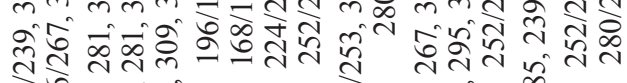
तె

응ㅎㅎ

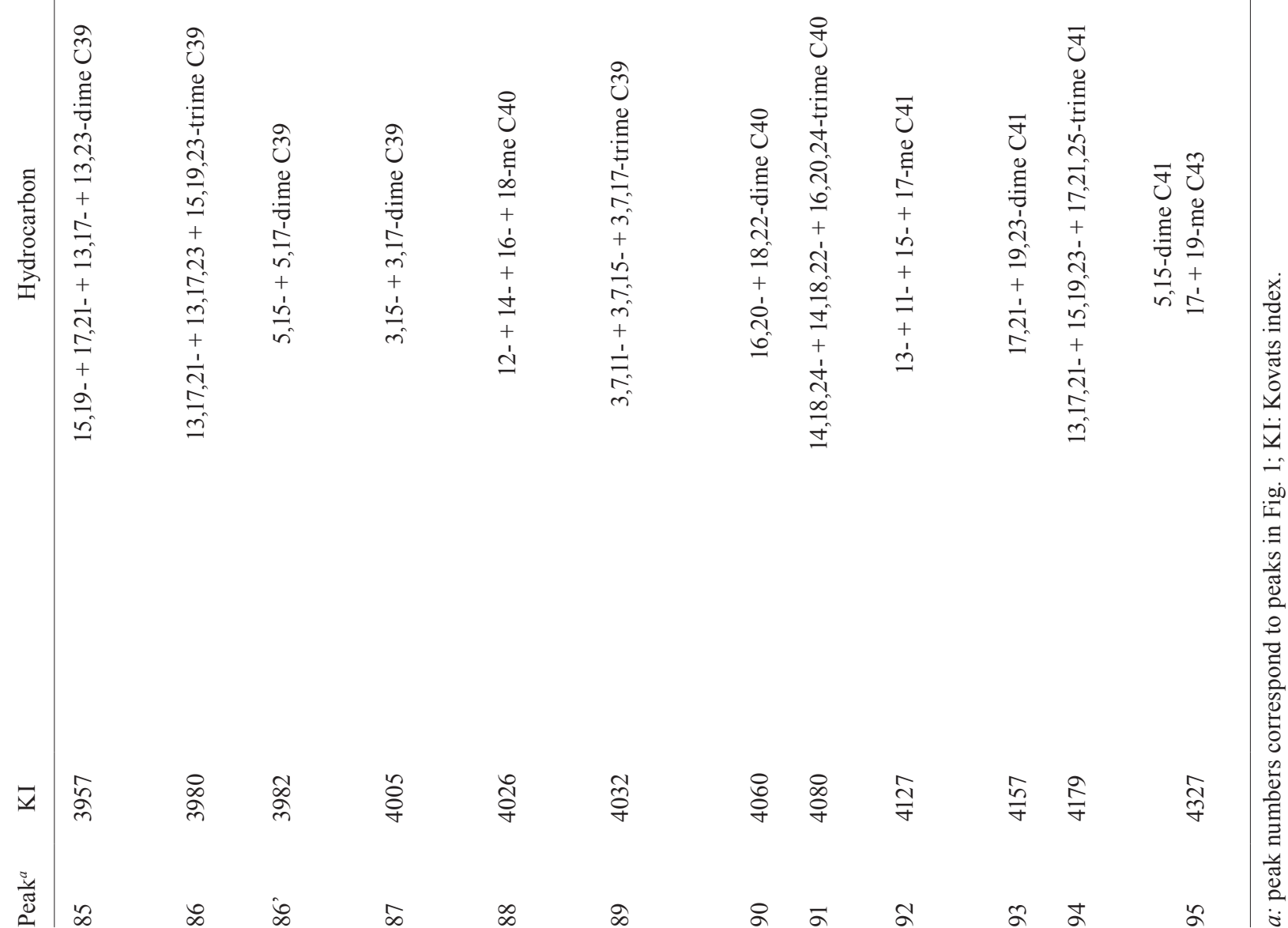




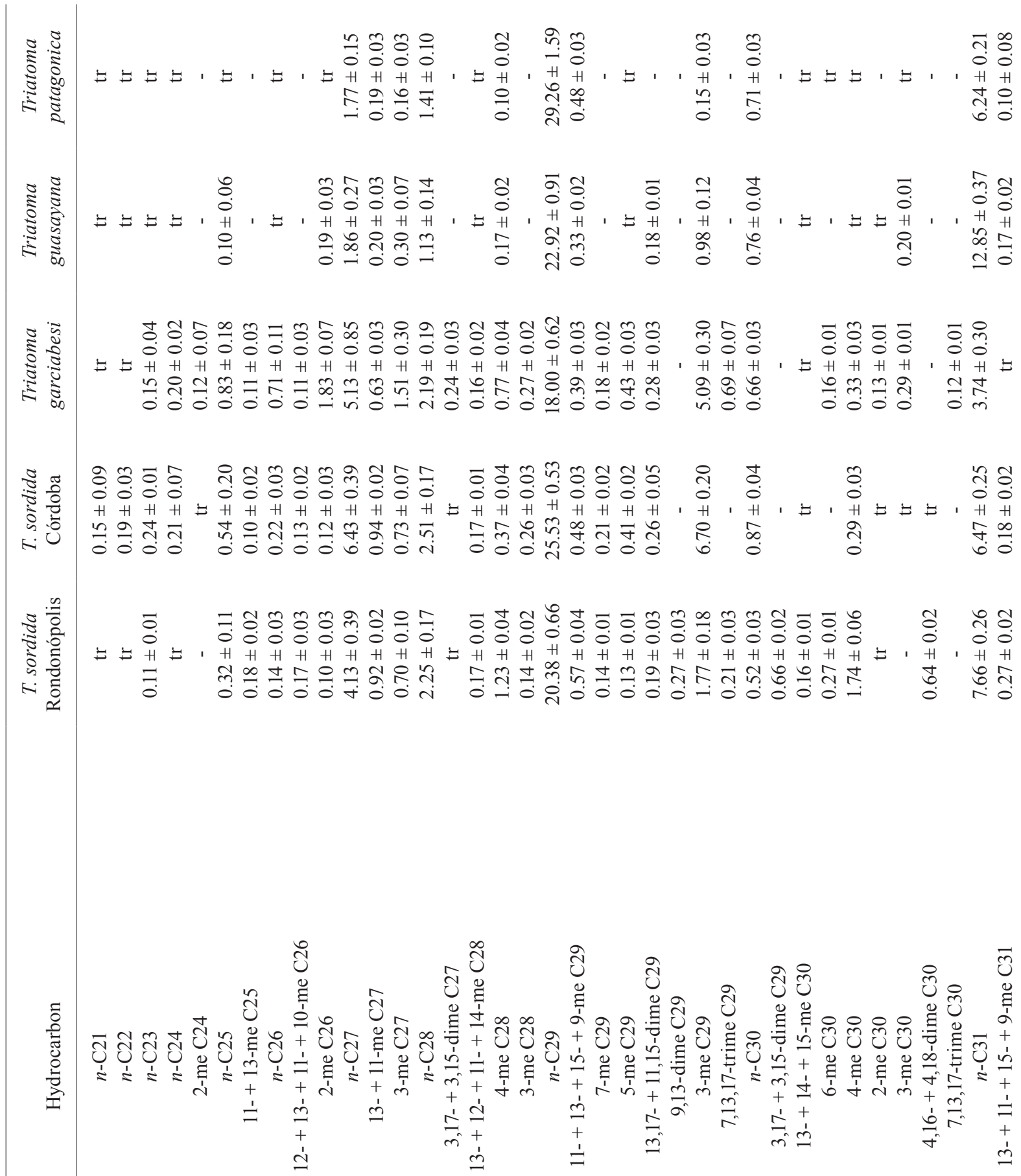




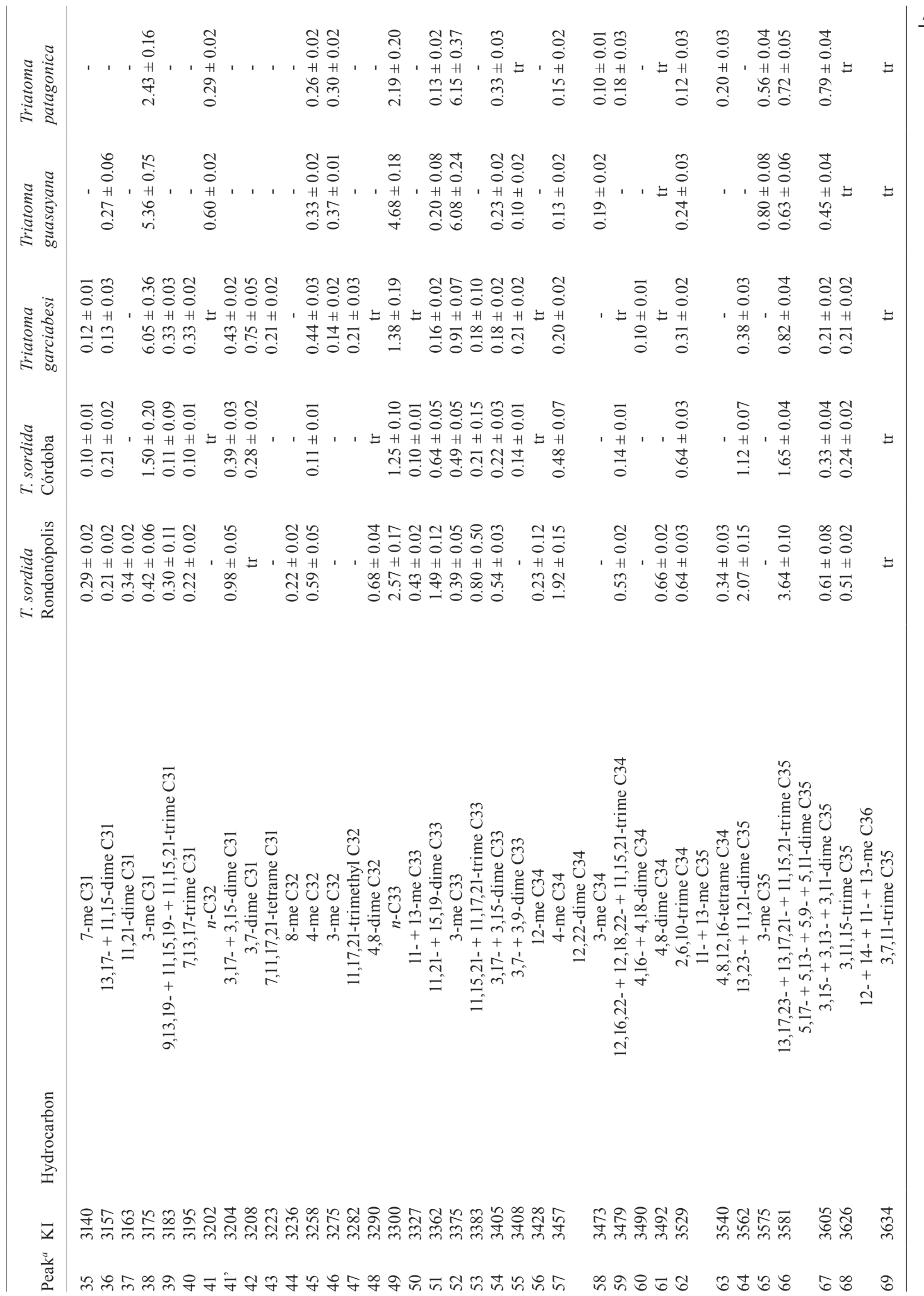




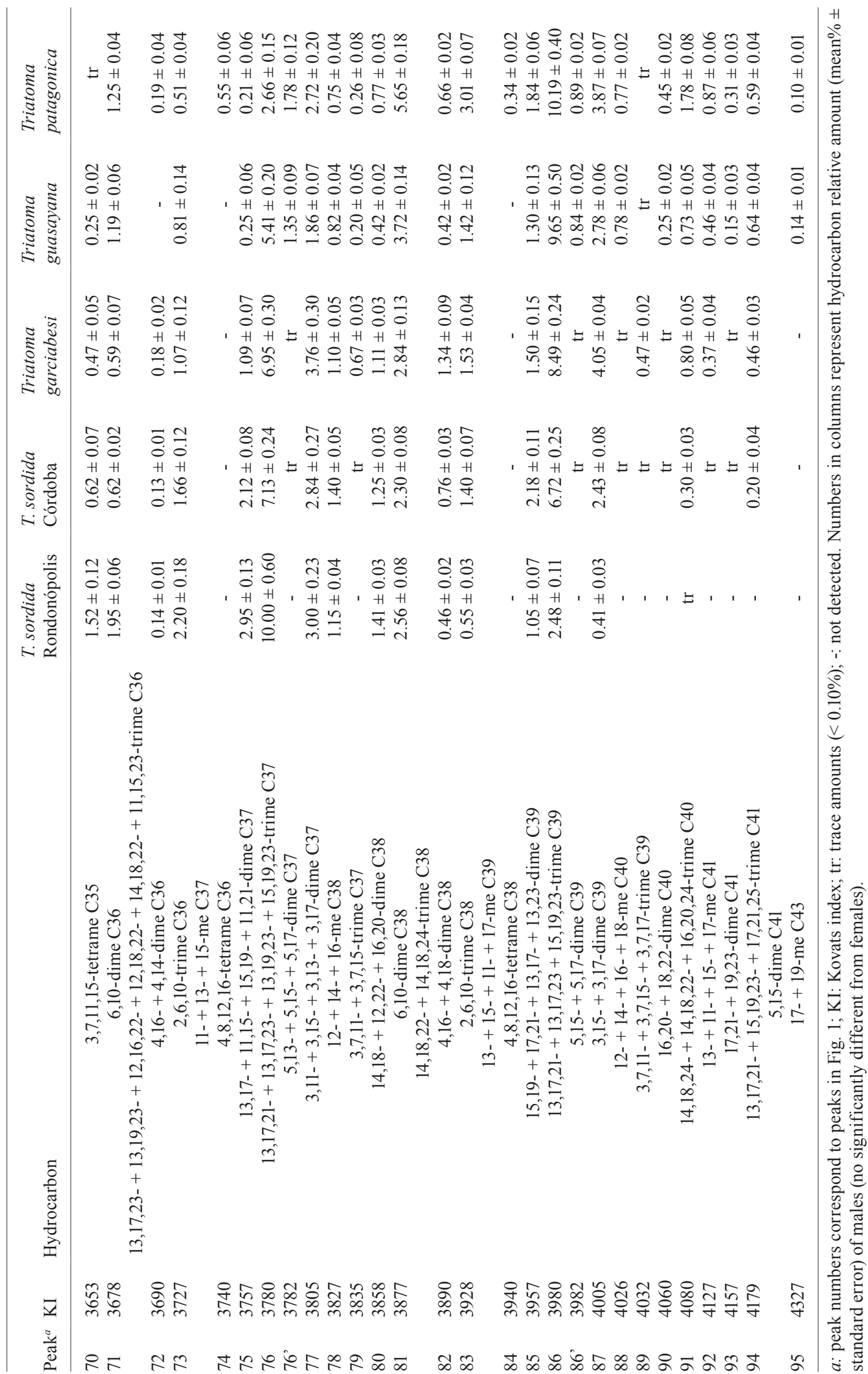

